\title{
Gripper robótico antropomórfico a los dedos primero y segundo, sensible a la presión
}

\author{
Francisco O. Gonzalez-Espinosa ${ }^{1}$, Erick D. de la Rosa-Montero ${ }^{1}$, \\ Carlos Rios-Ramirez ${ }^{1}$, Yesenia E. Gonzalez-Navarro ${ }^{2}$ \\ ${ }^{1}$ Instituto Politécnico Nacional, UPIITA, Academia de Biónica, \\ México \\ ${ }^{2}$ Instituto Politécnico Nacional, UPIITA, Academia de Sistemas, \\ México \\ ygonzalezn@ipn.mx
}

\begin{abstract}
Resumen. El presente trabajo aborda el diseño y construcción de un gripper robótico con cinco grados de libertad, capaz de realizar la prensión cilíndrica palmar y por oposición terminal de la mano humana. El gripper cuenta con la implementación de un sistema sensorial de presión donde cada eslabón posee un sensor con los cuales establece al contacto si hay o no un objeto en el espacio de trabajo del prototipo. La información de la posición final de los actuadores es utilizada en un algoritmo de control basado en reconocimiento de patrones con el cual el robot determina el tipo de agarre que realiza y la presión que debe de ejercer para sostener el objeto. Finalmente se activa el mecanismo de desplazamiento lineal que demuestra la capacidad del prototipo de sostener y asir el objeto.
\end{abstract}

Palabras clave: Gripper, sistema sensorial, presión, reconocimiento de patrones.

\section{Robotic Anthropomorphic Gripper of the First and Second Fingers Sensible to Pressure}

\begin{abstract}
This paper presented the designing and building of a robotic gripper with five degrees of freedom capable to perform cylindrical grasp and grasp by terminal opposition of the human hand. The gripper has a pressure sensorial system, where each link has a sensor to determine if exist contact with the object in the work space of the prototype. The final position information of the motors is using in the control algorithm of pattern recognition and this provides the information the grasp type that the robot is doing and the pressure that the robot must do to grasp and hold the objects. Finally the linear movement mechanism is activated to up.
\end{abstract}

Keywords: Gripper, sensorial system, pressure, patterns recognition. 


\section{Introducción}

Un gripper es un dispositivo electro-mecánico, cuya función es agarrar y sostener objetos específicos. Son comúnmente usados como actuadores finales de brazos robóticos o de prótesis de miembro superior. Con el fin de optimizar las tareas de agarre y sujeción de los robots, los elementos del gripper deben ser adaptables a la pieza de trabajo, esto es, sostener objetos con diferentes características, lo cual se logra maximizando el área de contacto entre el gripper y el elemento a sujetar, implementando distintos grados de libertad en el gripper [1], utilizando elementos flexibles pero firmes en los eslabones como el efecto derivado del movimiento de la cola de un pez [2] o con el uso de membranas flexibles que se pueden endurecer o ablandar mediante la modulación de aire dentro de ellas [3]. No obstante un robot capaz de aprender de su percepción y experiencia del entorno puede ajustarse a la características del objeto [4], lo cual se logra con la implementación de un sistema sensorial que comunique al actuador con su entorno y pueda distinguir entre las diferentes superficies de contacto, la presión ejercida y las fuerzas que intervienen [5].

Con las características mencionadas, a continuación este trabajo presenta el diseño y construcción del gripper; la sección 2 consiste en modelar la pinza del robot como los dedos primero y segundo de la mano humana, posteriormente las secciones 3 y 4 abordan el diseño y construcción mecánica y electrónica, finalmente en las secciones 5 y 6 se muestran los resultados de implementar el algoritmo de reconocimiento de patrones en el sistema de control del prototipo.

\section{Modelo matemático}

\subsection{Análisis robótico de la mano humana}

La mano humana es un gripper natural universal, la cual se analiza como un robot considerando cada una de las falanges de los dedos como los eslabones del mismo, unidas por juntas rotacionales que producen el movimiento de flexión y extensión o abducción y aducción.

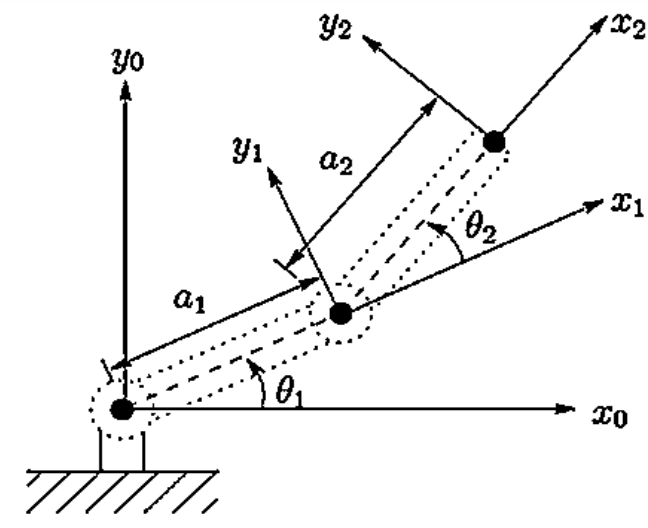

Fig. 1. Robot de dos grados de libertad. 
En este trabajo, el análisis se restringe al primer y segundo dedo de la mano humana, considerando solamente el movimiento de flexión y extensión de cada dedo y que cada uno posee dos falanges.

Se modela cada dedo como un robot de dos grados de libertad, donde el primer eslabón, el que se encuentra anclado al origen de un sistema cartesiano de coordenadas corresponde a la falange proximal para ambos dedos y el segundo eslabón corresponde a la falange distal del primer dedo y a la falange medial del segundo dedo, por lo tanto las variables incluidas en este modelo son: el ángulo entre sistemas coordenados $x_{0} \mathrm{y}$ $x_{1}\left(\theta_{1}\right)$; el ángulo entre sistemas coordenados $x_{1}$ y $x_{2}\left(\theta_{2}\right)$; la longitud del primer y segundo eslabón $a_{1}$ y $a_{2}$, ver Figura 1.

\subsection{Análisis cinemático}

El problema de la cinemática directa se refiere a la relación entre las articulaciones individuales del robot, la posición y la orientación del efector final [6]. Las variables de este análisis, mostradas en la Figura 1, son el conjunto de ángulos entre los eslabones en el caso de las juntas de rotación y la distancia entre eslabones en el caso de las articulaciones prismáticas. En la convención de Denavit Hartenberg cada eslabón del robot es representado por una matriz de transformación homogénea A, Ecuación (1), la cual está descrita por el producto de cuatro matrices básicas de transformación, rotación y traslación utilizando los ángulos de Euler, mostrada de forma explícita en la Ecuación (2). Finalmente el resultado del producto de todas las matrices A en su debido orden es la matriz de posición final T, Ecuación (3).

$$
\begin{gathered}
A=\operatorname{Rot}_{z, \theta} \cdot \operatorname{Tras}_{z, d} \cdot \operatorname{Tras}_{x, a} \cdot \operatorname{Rot}_{x, \alpha} \\
A=\left(\begin{array}{cccc}
\cos \theta & -\operatorname{sen} \theta & 0 & 0 \\
\operatorname{sen} \theta & \cos \theta & 0 & 0 \\
0 & 0 & 1 & 0 \\
0 & 0 & 0 & 1
\end{array}\right) \cdot\left(\begin{array}{cccc}
1 & 0 & 0 & d_{1} \\
0 & 1 & 0 & d_{2} \\
0 & 0 & 1 & d_{3} \\
0 & 0 & 0 & 1
\end{array}\right) \cdot\left(\begin{array}{cccc}
1 & 0 & 0 & a_{1} \\
0 & 1 & 0 & a_{2} \\
0 & 0 & 1 & a_{3} \\
0 & 0 & 0 & 1
\end{array}\right) \cdot\left(\begin{array}{cccc}
1 & 0 & 0 & 0 \\
0 & \cos \alpha & -\operatorname{sen} \alpha 0 \\
0 & \operatorname{sen} \alpha & \cos \alpha & 0 \\
0 & 0 & 0 & 1
\end{array}\right) \\
T=A_{1} \cdot A_{2} \cdot \ldots \cdot A_{n-1} \cdot A_{n}
\end{gathered}
$$

Siguiendo la convención de Denavit Hartenberg, cada dedo posee dos juntas. Por cada junta se tiene una matriz de transformación $A_{1}$ y $A_{2}$, Ecuación (4). Finalmente se tiene la expresión de posición del robot multiplicando la matriz $A_{i}$ correspondiente a cada junta del robot, donde el primer renglón corresponde a la falange proximal y el segundo la falange medial para cada dedo, Ecuación (5).

$$
\begin{gathered}
T=A_{1} \cdot A_{2}=\left[\begin{array}{cccc}
\cos \left(\theta_{1}\right) & -\operatorname{sen}\left(\theta_{1}\right) & 0 & a_{1} \cos \left(\theta_{1}\right) \\
\operatorname{sen}\left(\theta_{1}\right) & \cos \left(\theta_{1}\right) & 0 & a_{1} \cos \left(\theta_{1}\right) \\
0 & 0 & 1 & 0 \\
0 & 0 & 0 & 1
\end{array}\right] \cdot\left[\begin{array}{cccc}
\cos \left(\theta_{2}\right) & -\operatorname{sen}\left(\theta_{2}\right) & 0 & a_{2} \cos \left(\theta_{2}\right) \\
\operatorname{sen}\left(\theta_{2}\right) & \cos \left(\theta_{2}\right) & 0 & a_{2} \cos \left(\theta_{2}\right) \\
0 & 0 & 1 & 0 \\
0 & 0 & 0 & 1
\end{array}\right] \\
T=A_{1} \cdot A_{2}=\left[\begin{array}{cccc}
\cos \left(\theta_{1}+\theta_{2}\right) & -\sin \left(\theta_{1}+\theta_{2}\right) & 0 & a_{1} \cos \left(\theta_{1}\right)+a_{2} \cos \left(\theta_{1}+\theta_{2}\right) \\
\sin \left(\theta_{1}+\theta_{2}\right) & \cos \left(\theta_{1}+\theta_{2}\right) & 0 & a_{1} \sin \left(\theta_{1}\right)+a_{2} \sin \left(\theta_{1}+\theta_{2}\right) \\
0 & 0 & 1 & 0 \\
0 & 0 & 0 & 1
\end{array}\right]
\end{gathered}
$$




\subsection{Análisis dinámico}

El análisis dinámico del robot es de gran utilidad para calcular el torque necesario para cada actuador. Se realiza a partir de las ecuaciones de posición del mismo resultantes de la matriz de transformación $T$ y con las fuerzas que intervienen en él. La Ecuación (6) describe la ecuación general de torque.

$$
F=\sum_{i=1}^{n} F_{\text {rep }_{i}} \Omega+\sum_{i=1}^{n} F_{\text {att }_{i}} \Omega=\sum_{i=1}^{n} J_{a i}^{T} \Omega,
$$

donde $\Omega$ es la carga, $F_{\text {rep }}$ son las fuerzas repulsivas y $F_{\text {att }}$ son las fuerzas atractivas que intervienen y $J^{T}$ es el Jacobiano transpuesto de la ecuación de posición final de cada articulación del robot. Se calcula el Jacobiano de las funciones que se encuentran en la última columna de las matrices $A_{1}$ y $T$ respectivamente, Ecuaciones (7) y (8):

$$
\begin{gathered}
J_{A 1}=\left[\begin{array}{cc}
-a_{1} \operatorname{sen}\left(\theta_{1}\right) & 0 \\
a_{2} \cos \left(\theta_{2}\right) & 0
\end{array}\right] \\
J_{T}=\left[\begin{array}{cc}
-a_{1} \operatorname{sen}\left(\theta_{1}\right)-a_{2} \operatorname{sen}\left(\theta_{1}+\theta_{2}\right) & -a_{1} \operatorname{sen}\left(\theta_{1}+\theta_{2}\right) \\
a_{1} \cos \left(\theta_{1}\right)+a_{2} \cos \left(\theta_{1}+\theta_{2}\right) & a_{2} \cos \left(\theta_{1}+\theta_{2}\right)
\end{array}\right]
\end{gathered}
$$
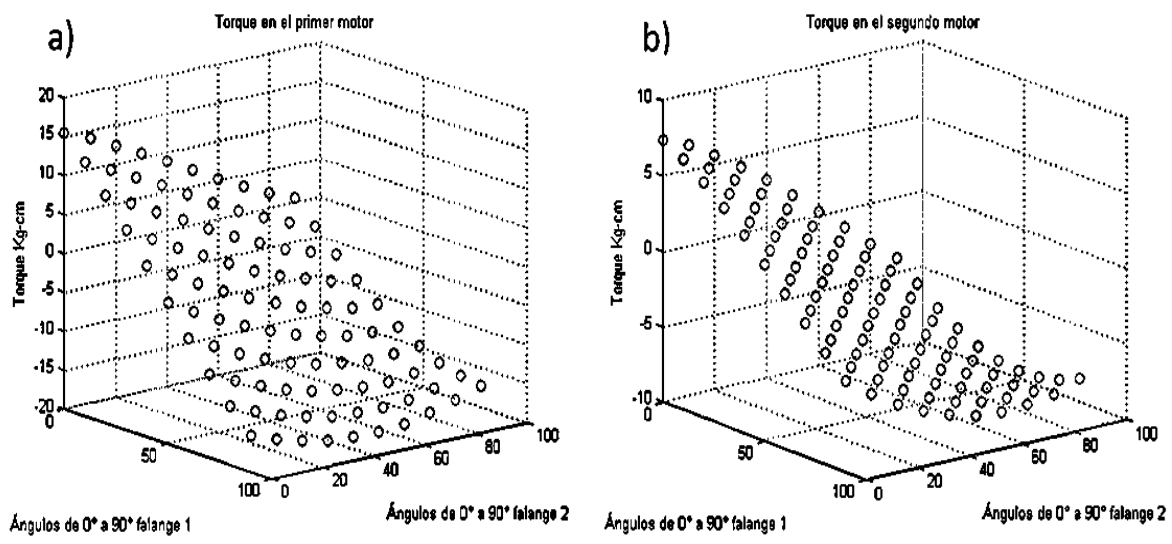

Fig. 2. Torque en los motores, a) primera falange, b) segunda falange.

Finalmente se realiza la suma de los productos de los Jacobianos transpuestos por el vector $\Omega$ que son las fuerzas que actúan en unidades Newton metro:

$$
F=\left[\begin{array}{c}
-2 l_{1} \sin \left(\theta_{1}\right)+2 l_{1} \cos \left(\theta_{1}\right) \Omega_{2}-l_{2} \sin \left(\theta_{1}+\theta_{2}\right)+l_{2} \cos \left(\theta_{1}+\theta_{2}\right) \Omega_{2} \\
-l_{1} \sin \left(\theta_{1}+\theta_{2}\right) \Omega_{1}+l_{2} \cos \left(\theta_{1}+\theta_{2}\right) \Omega_{2}
\end{array}\right]
$$

La Ecuación (9) se evalúa con un barrido de ángulos de $0^{\circ}$ a $90^{\circ}$ para cada articulación del gripper y en la Figura 2 se muestra el torque para cada posición que puede tomar cada motor correspondiente a las falanges, expresado en kilogramos por centímetro, con lo cual se seleccionaron motores con torque de $15 \mathrm{~kg}-\mathrm{cm}$. Para este 
cálculo se consideró la aplicación de una fuerza producida por una masa de dos kilogramos, también se incluye un coeficiente de fricción de 0.48 correspondiente al plástico ABS (Acrilonitrilo Butadieno Estireno) [7].

\section{Diseño y construcción mecánica}

\subsection{Análisis de esfuerzos y criterio de falla}

El diseño del gripper está íntimamente relacionado con las dimensiones de los motores y sensores que se implementaron. Este diseño se realizó en el software SolidWorks, Figura 3, con el cual se realizó un estudio de análisis de esfuerzos para cada una de las piezas que componen el gripper, con el fin de conocer la intensidad de las fuerza internas que actúan dentro del cuerpo, calcular las deformaciones del cuerpo y proveer un estudio de la estabilidad del mismo cuando está sometido a fuerzas externas, lo cual depende del tamaño de los miembros, sus deflexiones y su estabilidad dependen de las cargas internas y el material del que están hechas y utilizando la teoría del esfuerzo cortante máximo o criterio de Tresca, se predice el esfuerzo de falla de un material dúctil sometido a cualquier carga. En consecuencia para evitar la falla, el esfuerzo constante máximo debe ser menor o igual a la mitad de la resistencia a la tracción del material $\left(\sigma_{y} / 2\right)$ [8]. En la Tabla 1 se muestran los resultados de someter a las piezas del prototipo a la simulación de análisis de esfuerzos, considerando una fuerza de 20 Newtons, equivalente a más del doble de la fuerza a la cual será sometido el prototipo.

Tabla 1. Tensión máxima de Von Mises y factor de seguridad de los elementos del prototipo.

\begin{tabular}{lccc}
\hline \multicolumn{1}{c}{ Pieza } & $\begin{array}{c}\text { Tensión de Von } \\
\text { Mises Máxima }(\boldsymbol{N} / \\
\left.\mathbf{m}^{\mathbf{2}}\right)\end{array}$ & $\begin{array}{c}\text { Factor de } \\
\text { Seguridad Mínimo } \\
\text { (Tresca). }\end{array}$ & Nodo \\
\hline Base inferior & $3.89559 \mathrm{e}+006$ & 7.09025 & 15022 \\
Falange proximal 1 parte inferior & $1.97519 \mathrm{e}+007$ & 1.31537 & 15630 \\
Falange proximal 2 parte inferior & $1.87217 \mathrm{e}+007$ & 1.38977 & 106 \\
Falange distal 1 parte inferior & $1.82166 \mathrm{e}+007$ & 1.43566 & 70 \\
Falange distal 2 parte inferior & $1.79774 \mathrm{e}+007$ & 1.45655 & 70 \\
Eslabón corto de 5.5 cm & 705789 & 31.8458 & 125 \\
Eslabón largo de $8.5 \mathrm{~cm}$ & 313511 & 77.6142 & 11246 \\
\hline
\end{tabular}

a)

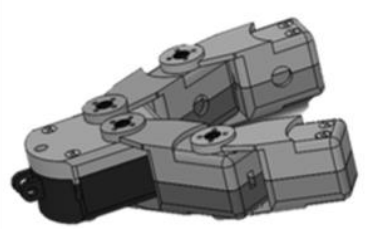

b)

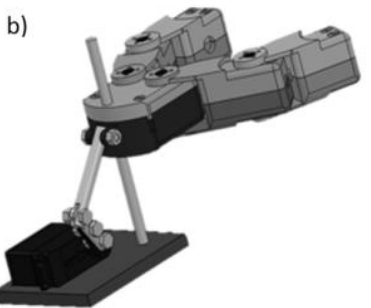

Fig. 3. Prototipo ensamblado, a) gripper completo, b) prototipo completo. 


\subsection{Maquinado e impresión 3D de los elementos constituyentes del prototipo}

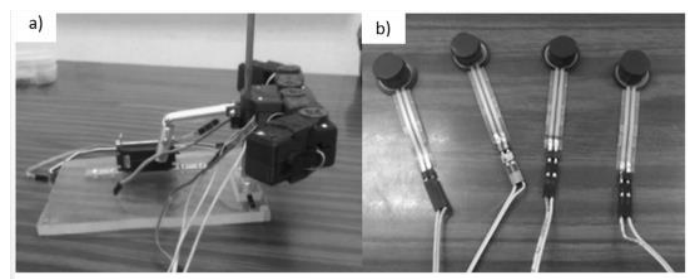

Fig. 4. Implementación, a) prototipo b) superficie de contacto en los sensores de presión.

La construcción de los elementos constituyentes del gripper se realizó mediante la impresión 3D. Por otro lado, los eslabones del mecanismo de desplazamiento lineal se maquinaron de forma convencional con ayuda del torno y la fresadora. En la Figura 4 se muestran los elementos maquinados, impresos y ensamblados. Además de la implementación del sistema sensorial, en el cual se coloca espuma de caucho en la superficie de contacto de cada sensor, brindando deformación al contacto.

\section{Diseño e instrumentación electrónica}

El prototipo es alimentado con tres baterías de 12 Volts a 2 amperios, las cuales son reguladas por tres fuentes conmutadas de bajada al voltaje de alimentación de los servomotores (6 volts a máximo 2 amperios). Están diseñadas con el circuito integrado LM2596 en la configuración recomendada por el fabricante. Con la finalidad de evitar algún corto circuito al conectar las baterías, se implementó un circuito sencillo con diodos e interruptores de encendido y apagado del prototipo.

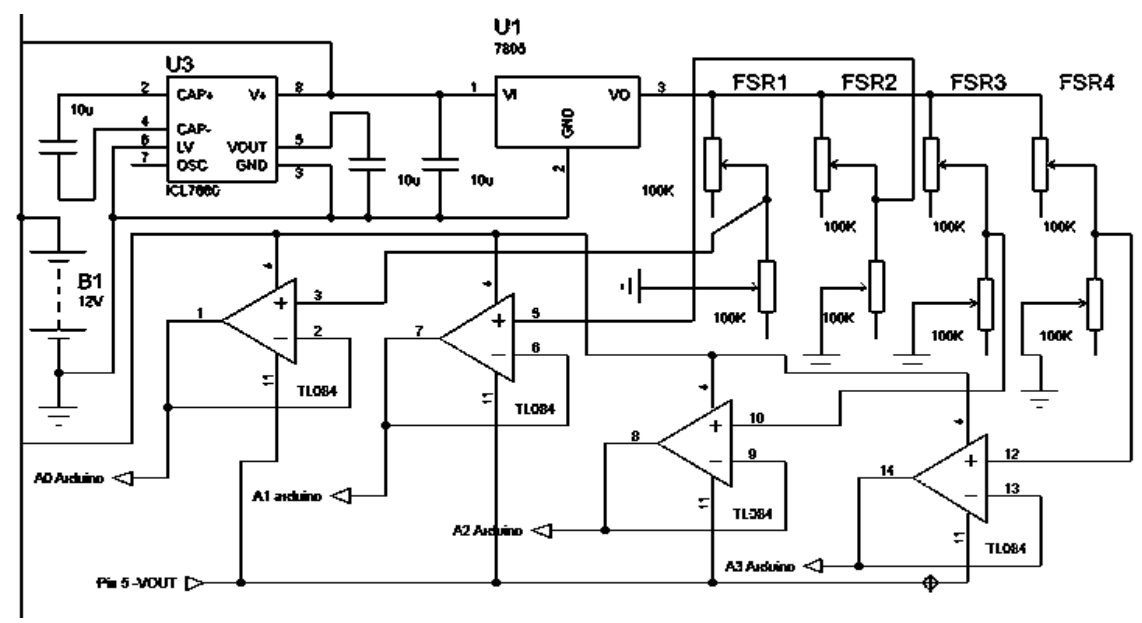

Fig. 5. Circuito completo de instrumentación de los sensores de presión.

El sensor de presión FSR tiene un comportamiento logarítmico que al incrementar la fuerza de aplicación en él, la resistencia disminuye [9]. Se usó la configuración 
recomendada por el fabricante en la que puede trabajar el sensor, en la cual el voltaje de salida es calculado por la Ecuación (10), que es un divisor de voltaje conectado a un seguidor de tensión construido con amplificadores operacionales, este último con el objetivo de acoplar impedancias y evitar la caída de tensión al conectarlo con el microcontrolador, el uso de amplificadores operacionales hace necesaria la inclusión de un circuito inversor de voltaje, matricula 7660, para evitar el uso de fuentes de tensión negativas de voltaje, el circuito electrónico completo de instrumentación de los sensores de presión se muestra en la Figura 5. Finalmente se utiliza un micro controlador ATMEGA328P en la etapa digital, debido a su implementación en la plataforma Arduino, el cual es el encargado de ser la unidad de lectura e interpretación de los sensores, realizar el reconocimiento de patrones y controlar los motores mediante modulación por ancho de pulso.

$$
V_{\text {salida }}=\frac{V_{+} R}{\left(R+R_{F S R}\right)}
$$

\section{Reconocimiento de patrones}

\subsection{Adquisición de patrones}

Para entrenar el algoritmo de reconocimiento de patrones previamente se deben extraer las características más sobresalientes de los objetos a clasificar, en este caso, estas características son la forma de los objetos que el gripper puede sostener, la diferencia entre la posición inicial y final de la falange proximal del primer dedo AFP1, la diferencia entre la posición inicial y final de la falange distal del primer dedo AFD1, la diferencia entre la posición inicial y final de la falange proximal del segundo dedo AFP2, la diferencia entre la posición inicial y final de la falange distal del segundo dedo AFD2, y la presión registrada por el sensor, ejercida entre el objeto y el gripper, Tabla 2. Esto se logra con el prototipo ensamblado con las etapas mecánica y electrónica en conjunto con la inclusión de un programa en el micro controlador que permite realizar la lectura de los sensores de presión y controlar la posición de los motores. Apoyado con un circuito que consta de cinco botones, los cuales envían señales de control al micro controlador, estas señales son: reinicio, activar ascenso del mecanismo, activar movimiento de los eslabones del gripper, activar interrupción 1, activar interrupción 2. Una vez que se enciende el prototipo, el gripper toma la posición inicial, se presiona el botón que activa el movimiento de las falanges proximales de forma simultanea hasta hacer contacto con el objeto y se presiona el botón de la interrupción 1 para detener el movimiento de las falanges proximales y comenzar con el movimiento de las falanges distales, posteriormente al hacer éstas contacto con el objeto se presiona el botón 2 para detener finalmente el movimiento de las falanges, se toma lectura de los valores arrojados por los sensores y la información es visualizada en la pantalla de una computadora al igual que la posición final de cada motor, se presiona el botón que acciona el mecanismo ascendente y se demuestra la sujeción del objeto. Se presiona el botón de reinicio, el gripper toma la posición inicial y repite el proceso. Los datos provenientes del sensor con los que se trabaja han sido discretizados en el intervalo de cero a 1024 . 
Francisco O. Gonzalez-Espinosa, Erick D. de la Rosa-Montero, Carlos Rios-Ramirez, et al.

Tabla 2. Diferencia en grados, posición de los motores.

\begin{tabular}{ccccccccc}
\hline \multicolumn{6}{c}{ Diferencias entre valores iniciales y finales de las características de los objetos } \\
\hline Objeto & AFP2 & AFD2 & AFP1 & AFD1 & SFP2 & SFD2 & SFP1 & SFD1 \\
Cartón & -89 & -66 & 89 & 66 & -4 & 282 & -4 & 96 \\
Tarjeta & -89 & -64 & 89 & 64 & 2 & 144 & 2 & 88 \\
Caja & -89 & -58 & 89 & 58 & -5 & 105 & -5 & 58 \\
Sintra 3 mm & -89 & -64 & 89 & 64 & -6 & 191 & -6 & 107 \\
Acrílico 1 cm & -89 & -76 & 89 & 76 & -5 & 344 & -5 & 308 \\
Botella 200 gr & -58 & -115 & 58 & 115 & 62 & -5 & 62 & 210 \\
Botella 1 kg & -70 & -125 & 70 & 125 & 11 & 130 & 111 & -21 \\
Tostada & -89 & -65 & 89 & 65 & -1 & -2 & -1 & 137 \\
Huevo & -45 & -75 & 45 & 75 & 2 & -2 & 2 & 82 \\
Cubo Rubik & -77 & -71 & 77 & 71 & -6 & -2 & -6 & 394 \\
\hline
\end{tabular}

\subsection{Diseño del algoritmo de reconocimiento de patrones}

Es necesario dividir el espacio de trabajo en cuatro regiones a clasificar que corresponden a la prensión por oposición terminal, prensión palmar cilíndrica, agarre rectangular y agarre irregular, Figura 6. Se agregan las dos últimas regiones debido a que el gripper por sus características mecánicas puede realizar estas prensiones, pero la mano humana no, por lo cual no pueden clasificarse en las regiones de prensión por oposición terminal y palmar cilíndrica, de forma que hacerlo podría generar un error en el sistema de control por solo tener dos regiones a clasificar en un amplio espacio de trabajo.
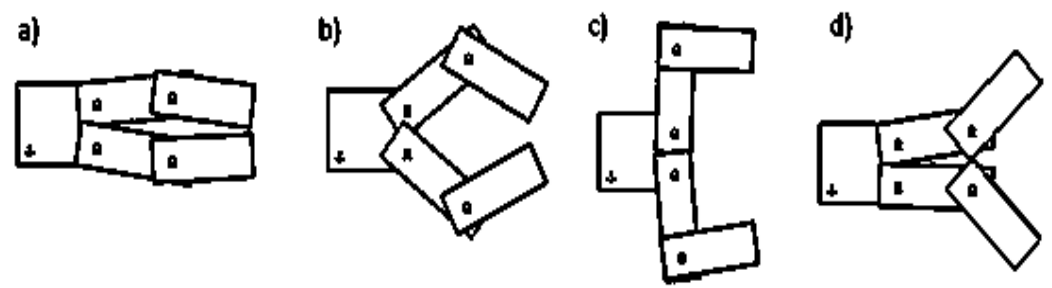

Fig. 6. Posiciones del gripper, a) prensión por oposición terminal, b) prensión palmar cilíndrica,

c) agarre rectangular, d) agarre irregular.

\subsection{Clasificador euclidiano modificado}

Este clasificador resulta de aplicar el cuadrado a la distancia euclidiana, distancia mínima entre un punto y otro, Ecuación (11), y despreciar los términos no discriminantes de la ecuación desarrollada, Ecuación (12) [10].

$$
D=\sqrt{\left|x_{1}-z_{1}\right|^{2}+\left|x_{2}-z_{2}\right|^{2}}
$$




$$
f(x)=X^{T} \cdot Z-\frac{1}{2} Z^{T} \cdot Z
$$

Cuando la distancia euclidiana calculada es mínima el valor de la Ecuación (12) es máximo. La función lineal que regionaliza el espacio de los patrones en dos clases separadas, Ecuación (13), es la diferencia entre dos patrones evaluados en la Ecuación (12). Siguiendo la convención de las Ecuaciones (14) y (15), el lugar geométrico de la función resultante es igual con cero, es la mediatriz del segmento que une los centroides de las dos clases.

$$
\begin{gathered}
f_{d}(x)=f_{1}(x)-f_{2}(x) \\
f_{d}(x)>0 \rightarrow f_{1}(x)>f_{2}(x) \\
f_{d}(x)<0 \rightarrow f_{1}(x)<f_{2}(x)
\end{gathered}
$$

Se clasifica en una clase a los resultados con signo positivo y en otra a los resultados con signo negativo. De igual manera para más de dos regiones, la clasificación se realiza con una combinación de resultados con signo positivo y negativo. A partir de los datos AFP2, AFD2, AFP1 y AFD1 de la Tabla 2, se calculó el valor absoluto y posteriormente el promedio de estos valores y después se incrementó o decremento este valor, evaluándolos en las Ecuaciones (12) y (13) hasta llegar a la separación de las regiones, prensión por oposición terminal, prensión palmar cilíndrica, agarre rectangular y agarre irregular, obteniendo los patrones de entrenamiento: [60, 10], [30, $30],[5,0]$ y $[60,-30]$, respectivamente, donde las ecuaciones finales son las siguientes.

$$
\begin{gathered}
f_{1}(x)=2\left[X_{1} X_{2}\right] \cdot\left[\begin{array}{l}
60 \\
10
\end{array}\right]-\left[\begin{array}{ll}
60 & 10
\end{array}\right] \cdot\left[\begin{array}{l}
60 \\
10
\end{array}\right]=120 X_{1}+20 X_{2}-3700 \\
f_{2}(x)=2\left[X_{1} X_{2}\right] \cdot\left[\begin{array}{l}
30 \\
30
\end{array}\right]-\left[\begin{array}{ll}
30 & 30
\end{array}\right] \cdot\left[\begin{array}{l}
30 \\
30
\end{array}\right]=60 X_{1}+60 X_{2}-1800 \\
f_{3}(x)=2\left[X_{1} X_{2}\right] \cdot\left[\begin{array}{l}
5 \\
0
\end{array}\right]-\left[\begin{array}{ll}
5 & 0
\end{array}\right] \cdot\left[\begin{array}{l}
5 \\
0
\end{array}\right]=10 X_{1}-25 \\
f_{4}(x)=2\left[X_{1} X_{2}\right] \cdot\left[\begin{array}{c}
60 \\
-30
\end{array}\right]-[60,-30] \cdot\left[\begin{array}{c}
60 \\
-30
\end{array}\right]=120 X_{1}-60 X_{2}-4500
\end{gathered}
$$

Se calculan las dos funciones discriminantes utilizando la Ecuación (13) entre $f_{1}(x)$ y $f_{2}(x)$ y entre $f_{2}(x)$ y $f_{3}(x)$.

$$
\begin{aligned}
& f_{12}(x)=f_{1}(x)-f_{2}(x)=60 X_{1}-40 X_{2}-1900 \\
& f_{23}(x)=f_{2}(x)-f_{3}(x)=50 X_{1}+60 X_{2}-1775
\end{aligned}
$$

\section{Implementación del sistema de control}

Utilizando MATLAB se graficaron las regiones del clasificador divididas por dos funciones discriminantes, Figura 7. La pendiente negativa resultado de entrenar el algoritmo con los patrones $[5,0]$ y $[30,30]$. La pendiente positiva es resultado de entrenar el algoritmo con los patrones $[60,10]$ y $[30,30]$. 

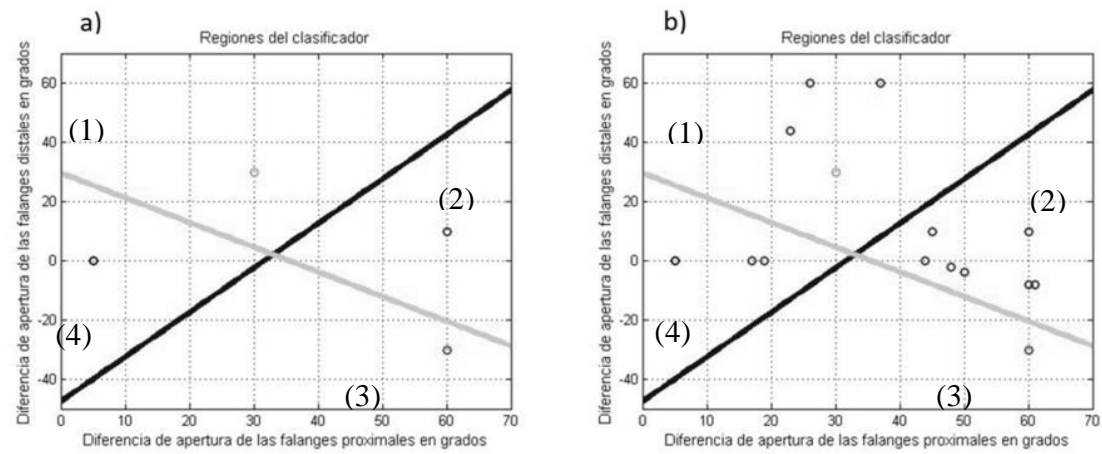

Fig. 7. Clasificador, a) Regiones, 1: cilíndrica, 2: plana, 3: irregular, 4: rectangular, b) Elementos clasificados.

Tabla 3. Resultados de la clasificación de distintos objetos.

\begin{tabular}{|c|c|c|c|c|c|}
\hline Objeto & $\begin{array}{c}\text { Resultado } \\
\text { Ecuación } \\
1\end{array}$ & $\begin{array}{c}\text { Resultado } \\
\text { Ecuación } 2\end{array}$ & Ángulo $1\left(^{\circ}\right)$ & Ángulo $2\left(^{\circ}\right)$ & $\begin{array}{c}\text { Tipo de } \\
\text { prensión }\end{array}$ \\
\hline Tarjeta & 2080 & 795 & 60 & -8 & O. Terminal \\
\hline $\begin{array}{l}\text { Botella de } \\
\text { aceite }\end{array}$ & 1260 & 485 & 50 & -4 & O. Terminal \\
\hline Cubo rubik & 740 & 425 & 44 & 0 & O. Terminal \\
\hline Tostada & 2080 & 795 & 61 & -8 & O. Terminal \\
\hline Huevo & 1060 & 505 & 48 & -2 & O. Terminal \\
\hline Pinzas & 380 & 125 & 38 & 0 & O. Terminal \\
\hline $\begin{array}{l}\text { Botella de } \\
200 \mathrm{ml}\end{array}$ & -2740 & 3125 & 26 & 60 & P. Cilíndrica \\
\hline $\begin{array}{l}\text { Botella de } \\
1 \mathrm{Lt}\end{array}$ & -2280 & 2015 & 23 & 44 & P. Cilíndrica \\
\hline $\begin{array}{l}\text { Botella de } \\
600 \mathrm{ml}\end{array}$ & -2080 & 3675 & 37 & 60 & P. Cilíndrica \\
\hline $\begin{array}{l}\text { Eje de robot } \\
\text { serpiente }\end{array}$ & -76 & -825 & 19 & 0 & Rectangular \\
\hline Multímetro & -880 & -925 & 17 & 0 & Rectangular \\
\hline
\end{tabular}

El algoritmo de control desarrollado sobre la plataforma Arduino, coloca al gripper en una posición inicial y se mantiene hasta recibir la señal de activación, después los motores que corresponden a las falanges proximales comienzan a moverse simultáneamente de modo que la apertura entre una y otra disminuye y realiza la lectura de todos los sensores de presión. Posteriormente existen dos escenarios. El primero si alguno de los sensores de presión que se encuentran en las falanges proximales detecta variación, se detendrán los motores activando los motores correspondientes a las falanges distales, los cuales se mueven de igual forma que los anteriores, disminuyendo 
la apertura entre ellos hasta que uno de los sensores colocados en estas falanges detecte algo o bien se toquen entre ellas.

El segundo escenario sucede cuando los sensores de presión colocados en las falanges proximales no detectan nada y se llega a una distancia mínima entre ellos provocando que los motores en las falanges distales se muevan como el caso anterior. Una vez que los motores del gripper se han detenido, la posición actual en grados de cada uno es evaluada en el algoritmo de reconocimiento de patrones y éste determina el tipo de prensión que realiza el gripper. Dependiendo del resultado, el gripper disminuye la distancia entre las falanges proximales y entre las falanges distales de modo que la presión entre el objeto y el gripper aumenta hasta llegar a un umbral, el cual es distinto para cada región. Finalmente se activa el mecanismo de desplazamiento lineal deslizando el gripper hacia arriba y lo mantiene hasta que llega una nueva señal de activación que deshace el agarre. Se probó el prototipo ensamblado y con el algoritmo de reconocimiento de patrones implementado en el sistema de control, obteniendo los resultados mostrados en la Figura 7 y la Tabla 3.

\section{Conclusiones}

Se desarrolló el modelo matemático de un gripper robótico antropomórfico a los dedos primero y segundo, analizando a cada dedo como un robot de dos grados de libertad, lo cual permite ubicar al robot en cualquier punto en su espacio de trabajo, además el modelo sirvió de base para el análisis dinámico del robot, lo que permitió conocer el torque necesario para cada motor. La respuesta logarítmica del sensor de presión contribuye al correcto funcionamiento del prototipo ya que cuando el sensor es sometido a una presión inicial se genera un cambio de valor con respecto al inicial, lo cual sirve para determinar de forma sencilla que el sensor entró en contacto con un objeto y que este cambio no es generado por ruido o interferencia. Se llevaron a cabo distintas pruebas de agarre y clasificación del sistema con objetos de distintas formas y pesos, presentando resultados satisfactorios. Una mejora al prototipo es que esté apoyado de un sistema de visión artificial el cual le indique dónde se encuentra el objeto y busque la mejor posición en que pueda sostenerlo.

\section{Referencias}

1. Cheraghpour, F., Feizollahi, A.: Design, Fabrication and Control of a Three Finger Robotic Gripper. In: First International Conference on Robot, Vision and Signal Processing, pp. 280-283 (2011)

2. FESTO, Adaptative gripper DHDG. pp. 1-4 (2011)

3. Jiang, Y., Amed, J.R., Lipson, H., Saxena, A.: Learning hardware agnostic grasps for a universal jamming gripper. In: IEEE International Conference on Robotics an automation, pp. 2385-2391 (2012)

4. Jagannathan, S., Galna, G.: Adaptative Critic Neural Network Based Object Grasping Control Using a Three Finger Gripper. IEEE Transactions on neural networks, Vol 15, No. 2, pp. 395-407 (2004) 
Francisco O. Gonzalez-Espinosa, Erick D. de la Rosa-Montero, Carlos Rios-Ramirez, et al.

5. Sun, L., Shan, J.H., Meng, M., Zhang, D., Mei, T.: Application of Intelligent Flexible Skin Sensors for Interfacing with Robotic Pets. In: First IEEE International Conference on Nano/Micro Engineered and Molecular Systems, pp. 1527-1531 (2006)

6. Spong, M.W., Hutchinson, S., Vidyasagar, M.: Forward and inverse kinematics: Robot Modeling and Control. John Wiley and Sons, pp. 75-186 (2005)

7. bove-ag Plásticos y elastómeros. http://www.plasticosmecanizables.com/plasticos _coeficiente_friccion.html

8. Hibeler, R.C.: Transformación de deformación unitaria: Mecánica de Materiales. Pearson, pp. 542-545 (2006)

9. Interlink Electronics. http://www.interlinkelectronics.com/datasheets/ Datasheet_ FSR.pdf

10. Theodoridis, S., Koutroumbas, K.: Classifiers Based on Bayes Decision Theory. Pattern Recognition, Elsevier, pp. 30-34 (2009) 